\title{
LS-CATÉGORIE DE CW-COMPLEXES À 3 CELLULES EN THÉORIE HOMOTOPIQUE $R$-LOCALE
}

\author{
Hans Scheerer et Daniel Tanré
}

\begin{abstract}
We study the Lusternik-Schnirelmann category of some CW-complexes with 3 cells, built on $Y=S^{2 n} \cup_{k\left[\iota_{2 n}, \iota_{2 n}\right]} e^{4 n}$. In particular, we prove that an $R$-local space, in the sense of D. Anick, of LS-category 3 and of the homotopy type of a CW-complex with $3 R$-cells, has a cup-product of length 3 in its algebra of cohomology. This result is no longer true in the framework of mild spaces.
\end{abstract}

La LS-catégorie d'un espace $X$, cat $X$, a été définie par Lusternik et Schnirelmann, [10], en 1934, pour minorer le nombre de points critiques d'une variété compacte, sans bord. Elle s'est révélée être un invariant homotopique difficile à déterminer et son comportement lors de l'attachement d'une cellule est un problème ouvert.

Dans [4], Berstein et Hilton caractérisent les CW-complexes à deux cellules de LS-catégorie 2. Nous nous intéressons ici à certains CW-complexes, de LS-catégorie 3, admettant une décomposition en 3 cellules, (éventuellement localisées). Des résultats existent déjà dans cette direction, [14], [15]. Ils concernent les espaces s'écrivant comme espace total d'un fibré de base et fibre une sphère; notons que la dualité de Poincaré s'y révèle une propriété fondamentale pour l'argumentation utilisée. La situation envisagée dans ce travail est différente; nous y étudions des CW-complexes, $Y \cup e^{p}$, obtenus en attachant une cellule à $Y=S^{2 n} \cup_{k\left[\iota_{2 n}, \iota_{2 n}\right]} e^{4 n}$, (où $\iota_{j}$ dénote l'application identité de $S^{j}$ et [, ] le crochet de Whitehead). On remarque que $Y$ est la cofibre, $\psi: M \vee S^{2 n} \longrightarrow Y$, d'une application $\gamma: S^{4 n-1} \longrightarrow M \vee S^{2 n}$, où $M$ est l'espace de Moore $M=S^{4 n-1} \cup_{k \iota_{4 n-1}} e^{4 n}$. Dans un premier résultat, nous déterminons l'image de $\Omega \psi$ :

Keywords. Lusternik-Schnirelmann cathegory, CW-complex.

1991 Mathematics subject classifications: 55P50, 55P62. 
Théorème 1. Une fois localisé en dehors de 2 et de 3, l'espace des lacets $\Omega Y$ admet une décomposition $\Omega Y=S^{2 n-1} \times \Omega S^{6 n-1} \times \Omega Y^{\prime}$, telle que l'application $\Omega \psi$ suivie de la projection $\Omega Y \rightarrow S^{2 n-1} \times \Omega Y^{\prime}$ ait un inverse à droite.

Ainsi, toute application $f: S^{p-1} \rightarrow Y$, dont l'adjointe $\tilde{f}: S^{p-2} \rightarrow \Omega Y$ est à valeurs dans $S^{2 n-1} \times \Omega Y^{\prime}$ se relève à travers $\psi$; i.e. il existe $f^{\prime}$ : $S^{p-1} \rightarrow M \vee S^{2 n}$ tel que $f=\psi \circ f^{\prime}$. Dans ce cas, l'espace $Y \cup_{f} e^{p}$ a le type d'homotopie de $\left(M \vee S^{2 n}\right) \cup_{\gamma} e^{4 n} \cup_{f^{\prime}} e^{p}$; il est de longueur en cône et de LS-catégorie $\leq 2$.

Pour que l'application $f: S^{p-1} \rightarrow Y$ donne naissance à un espace $Y \cup_{f}$ $e^{p}$ de LS-catégorie 3, il faut donc que l'application induite en homotopie par $\tilde{f}$ ait une composante non nulle en $\pi_{*}\left(\Omega S^{6 n-1}\right)$ dans la décomposition $\pi_{*}(\Omega Y)=\pi_{*}\left(S^{2 n-1}\right) \oplus \pi_{*}\left(\Omega S^{6 n-1}\right) \oplus \pi_{*}\left(\Omega Y^{\prime}\right)$. Pour les espaces $R$-locaux d'Anick, [2], [3], [5], [13], nous montrons que ceci implique l'existence d'un cup-produit de longueur 3 dans l'algèbre de cohomologie:

Soit $R$ un sous-anneau de $\mathbb{Q}$ et soit $r$ un entier fixé, $r \geq 3$, nous notons $\bar{p}$ le plus petit entier non inversible dans $R$ et posons $m:=r+2 \bar{p}-4$. Rappelons qu'une sphère $R$-locale, $S_{R}^{n}, n \geq 1$, est un CW-complexe $n$-réduit, abélien, vérifiant $H_{i}\left(S_{R}^{n}\right)=0, i \neq 0, n$ et $H_{n}\left(S_{R}^{n}\right) \cong R$. Un $\mathrm{CW}$-complexe $R$-local, $r$-réduit de $R$-dimension $m$ est un complexe cellulaire construit à partir d'un point par des attachements successifs de cônes sur des sphères $R$-locales, $S_{R}^{n}, r-1 \leq n<m$.

Notons $C W_{r}^{m}$ la catégorie des $C W$-complexes $R$-locaux, $r$-réduits et de $R$-dimension $\leq m$.

Théorème 2. Si $X \in C W_{r}^{m}$ est un $C W$-complexe de LS-catégorie 3 , admettant une décomposition en 3 cellules $R$-locales, alors son algèbre de cohomologie admet un cup-produit de longueur 3.

Le résultat énoncé ci-dessus devient évidemment faux pour des espaces plus généraux que les espaces $R$-locaux, comme le montre l'exemple de $S p(2)$, [14]. Remarquons cependant que, si on localise $S p(2)$ en dehors de 2 et de 3 , cadre du Théorème 1, alors l'espace obtenu a une LS-catégorie égale à la longueur du cup-produit, à savoir 2. Se pose donc la question de l'existence d'un contre-exemple au résultat du Théorème 2 faisant intervenir un espace localisé en dehors de 2 et de 3. C'est l'objet de la dernière section; nous remarquerons également que cet exemple est un espace tempéré (mild space) au sens d'Anick, [2]. 


\section{LS-catégorie}

Les "espaces" considérés dans cette section sont des espaces pointés ayant le type d'homotopie pointée d'un CW-complexe connexe. Cette restriction, cohérente avec la suite, simplifie la présentation de la LS-catégorie. Pour tout complément, nous renvoyons le lecteur aux deux articles de synthèse, $[\mathbf{7}],[\mathbf{9}]$.

Commençons par rappeler la définition de LS-catégorie sous une forme similaire à celle utilisée par Lusternik et Schnirelmann dans [10]:

Définition 3. La LS-catégorie, cat $X$, d'un espace $X$ est le plus petit entier $k, k \geq 0$, pour lequel $X$ peut-être recouvert par $(k+1)$ ouverts, contractiles dans $X$.

Notons que les conventions diffèrent suivant les travaux. Ici, nous attribuons la valeur 0 à la LS-catégorie d'un espace contractile, (dans de nombreux articles, [7], elle est égale à 1 ).

Ganea a montré que cette définition équivaut à l'existence de sections dans une suite de fibrations construites inductivement. Pour $F_{0} \rightarrow E_{0}(X) \rightarrow X$, on choisit la fibration universelle des chemins sur $X$, $\Omega X \rightarrow P X \rightarrow X$. Ensuite, à partir de la fibration

$$
F_{n} \rightarrow E_{n}(X) \stackrel{p_{n}}{\longrightarrow} X
$$

on construit $E_{n+1}(X)$ comme la cofibre de $F_{n} \rightarrow E_{n}(X), E_{n+1}(X)=$ $E_{n}(X) \cup_{F_{n}} C\left(F_{n}\right)$. L'espace $F_{n+1}$ est la fibre homotopique de l'application $p_{n+1}: E_{n+1}(X) \rightarrow X$, obtenue en envoyant le cône, $C\left(F_{n}\right)$, sur le point de base de $X$.

Proposition 4 [6]. La LS-catégorie, cat X, d'un espace $X$ est le plus petit entier $k, k \geq 0$, pour lequel la fibration $p_{k}: E_{k}(X) \rightarrow X$ admet une section homotopique, i.e. il existe $\sigma_{k}: X \rightarrow E_{k}(X)$, tel que $p_{k} \circ \sigma_{k} \simeq \operatorname{id}_{X}$.

Rappelons également, [6], que la LS-catégorie est majorée par la longueur en cône, $C a t X$, d'un espace:

Définition 5. Soit $X$ un espace, on pose:

- Cat $X=0$ si $X$ est contractile;

- Cat $X \leq k+1$ s'il existe une cofibration entre CW-complexes, $A \rightarrow B \rightarrow C$, telle que $B$ soit connexe, $C a t B \leq k$ et $C$ du type d'homotopie de $X$.

Dans toute la suite, nous confondrons application et classe d'homotopie d'application. 


\section{Démonstration du Théorème 1}

Remarquons d'abord que $Y$ s'obtient comme cofibre:

$$
S^{4 n-1} \stackrel{\varphi_{1}-\varphi_{2}}{\longrightarrow} M \vee S^{2 n} \stackrel{\psi}{\longrightarrow} Y=S^{2 n} \cup_{k\left[\iota_{2 n}, \iota_{2 n}\right]} e^{4 n}
$$

où:

- $M$ est l'espace de Moore $M=S^{4 n-1} \cup_{k \iota_{4 n-1}} e^{4 n}$;

- $\varphi_{1}: S^{4 n-1} \hookrightarrow M \hookrightarrow M \vee S^{2 n}$ est la composition des injections canoniques;

- $\varphi_{2}: S^{4 n-1} \rightarrow S^{2 n} \hookrightarrow M \vee S^{2 n}$ est la composition de l'injection canonique avec le crochet de Whitehead $\left[\iota_{2 n}, \iota_{2 n}\right]: S^{4 n-1} \rightarrow S^{2 n}$;

- $\varphi_{1}-\varphi_{2}$ s'obtient à partir de la structure usuelle de co-H espace de $S^{4 n-1}$.

Proposition 6. Nous supposons les espaces localisés en dehors de 2 et de 3. L'espace des lacets $\Omega Y$ admet une décomposition

$$
\Omega Y=S^{2 n-1} \times \Omega\left(M \vee S^{6 n-1}\right)
$$

Remarque. Avec une méthode légèrement différente, Neisendorfer et Selick, [12], établissent cette décomposition de $\Omega Y$ pour les $p$-localisés de $Y, p>3$.

Démonstration de la Proposition 6: La démonstration utilise le modèle introduit par Adams et Hilton pour "représenter" l'espace des lacets d'un CW-complexe; nous renvoyons le lecteur à $[\mathbf{1}]$ et $[\mathbf{8}]$ pour la justification des propriétés suivantes:

- Le modèle d'Adams Hilton est une algèbre tensorielle différentielle graduée construite par induction suivant les attachements cellulaires. Si $Z^{\prime}=Z \cup_{f} e^{n}$, avec $f: S^{n-1} \rightarrow Z$, le modèle $(T(W), \partial)$ de $Z^{\prime}$ s'obtient à partir du modèle $(T(V), \partial)$ de $Z$, en ajoutant à $V$ un générateur $\alpha$ de degré $n-1$ dont la différentielle $\partial \alpha$ est un cycle de $(T(V), \partial)$ représentant $H_{*}(\Omega f)\left(h_{n-2}\right)$, où $h_{n-2}$ est l'image du générateur de $H_{n-2}\left(S^{n-2}\right)$ par l'application $S^{n-2} \rightarrow \Omega S^{n-1}$, adjointe de $\iota_{n-1}$.

- Si $(T(V), \partial)$ est le modèle d'Adams Hilton de $Z$, l'application de complexes de chaînes, $(T(V), \partial) \otimes(T(V), \partial) \rightarrow(T(V), \partial)$, $w_{1} \otimes w_{2} \mapsto w_{1} w_{2}$, induit la structure d'algèbre de Pontrjagin de $H_{*}(\Omega Z)$. De même, le commutateur de $H_{*}(\Omega Z)$ est induit par l'application $w_{1} \otimes w_{2} \mapsto w_{1} w_{2}-(-1)^{\left|w_{1}\right|\left|w_{2}\right|} w_{2} w_{1}$. 
(1): Mise en évidence de $\tilde{\varphi}: S^{6 n-1} \rightarrow Y$.

Soit $M=\Sigma M^{\prime}=\Sigma\left(S^{4 n-2} \cup_{k \iota_{4 n-2}} e^{4 n-1}\right)$, nous notons:

- $j_{1}: M^{\prime} \rightarrow \Omega \Sigma M^{\prime} \rightarrow \Omega \Sigma\left(M^{\prime} \vee S^{2 n-1}\right)$,

- $j_{2}: S^{2 n-1} \rightarrow \Omega \Sigma S^{2 n-1} \rightarrow \Omega \Sigma\left(M^{\prime} \vee S^{2 n-1}\right)$,

les flèches obtenues avec l'adjonction $(\Omega, \Sigma)$ et les injections canoniques. Le commutateur pour la loi de $H$-espace est noté:

$$
\langle,\rangle: \Omega\left(M \vee S^{2 n}\right) \times \Omega\left(M \vee S^{2 n}\right) \rightarrow \Omega\left(M \vee S^{2 n}\right)
$$

L'application composée

$$
M^{\prime} \times S^{2 n-1} \stackrel{j_{1} \times j_{2}}{\longrightarrow} \Omega\left(M \vee S^{2 n}\right) \times \Omega\left(M \vee S^{2 n}\right) \stackrel{\iota,\rangle}{\longrightarrow} \Omega\left(M \vee S^{2 n}\right)
$$

a une restriction à $M^{\prime} \vee S^{2 n-1}$ homotopiquement triviale; elle induit donc une application

$$
\left\langle j_{1}, j_{2}\right\rangle: M^{\prime} \wedge S^{2 n-1} \rightarrow \Omega\left(M \vee S^{2 n}\right) .
$$

Le composé $S^{4 n-2} \wedge S^{2 n-1} \rightarrow M^{\prime} \wedge S^{2 n-1} \stackrel{\left\langle j_{1}, j_{2}\right\rangle}{\longrightarrow} \Omega\left(M \vee S^{2 n}\right) \stackrel{\Omega \psi}{\longrightarrow} \Omega Y$ s'écrit $\Omega \psi\left\langle\tilde{\varphi}_{1}, j_{2}\right\rangle$, où $\tilde{\varphi}_{1}$ est l'adjointe de $\varphi_{1}$; il est égal à $\Omega \psi\left\langle\tilde{\varphi}_{2}, j_{2}\right\rangle$, car $\Omega \psi\left(\tilde{\varphi}_{1}-\tilde{\varphi}_{2}\right)=0$. Remarquons que $\Omega \psi\left\langle\tilde{\varphi}_{2}, j_{2}\right\rangle$ est le composé de l'adjoint de $\left[\iota_{2 n},\left[\iota_{2 n}, \iota_{2 n}\right]\right]$ suivi de $\Omega S^{2 n} \longrightarrow \Omega\left(M \vee S^{2 n}\right)$. L'entier 3 étant inversé, le crochet de Whitehead itéré est homotopiquement trivial et l'application $\Omega \psi\left\langle j_{1}, j_{2}\right\rangle$ s'étend à la cofibre de $S^{4 n-2} \wedge S^{2 n-1} \rightarrow M^{\prime} \wedge$ $S^{2 n-1}$ en une application

$$
\varphi: S^{6 n-2} \cong S^{4 n-1} \wedge S^{2 n-1} \rightarrow \Omega Y .
$$

Un modèle d'Adams Hilton de $M^{\prime} \wedge S^{2 n-1}$ est $(T(\tilde{\alpha}, \alpha), \partial),|\alpha|=$ $|\tilde{\alpha}|+1=6 n-2 ; \partial \alpha=k \tilde{\alpha} ;$ un modèle de $Y$ est donné par $(T(a, b), \partial)$; $|a|=2 n-1 ;|b|=4 n-1 ; \partial b=k[a, a]$. L'application $(T(\tilde{\alpha}, \alpha), \partial) \rightarrow$ $(T(a, b), \partial)$, qui correspond à l'adjoint de $\Omega \psi\left\langle j_{1}, j_{2}\right\rangle$, applique $\tilde{\alpha}$ sur zéro et $\alpha$ sur $[a, b]$. Par conséquent, le modèle de l'adjoint $\tilde{\varphi}: S^{6 n-1} \rightarrow Y$ de $\varphi$ est le suivant:

$$
\mathcal{A}(\tilde{\varphi}):(T(\alpha), 0) \rightarrow(T(a, b), \partial) ;|\alpha|=6 n-2 ; \mathcal{A}(\tilde{\varphi})(\alpha)=[a, b] .
$$

(2): $\Omega Y$ a le type d'homotopie de $S^{2 n-1} \times \Omega\left(M \vee S^{6 n-1}\right)$.

Notons:

- $g_{1}: S^{2 n-1} \rightarrow \Omega Y$, l'adjointe de $S^{2 n} \hookrightarrow M \vee S^{2 n} \stackrel{\psi}{\rightarrow} Y$;

- $g_{2}=\tilde{\varphi}: S^{6 n-1} \rightarrow Y$, l'application construite dans (1);

- $g_{3}: M \rightarrow Y$, égale au composé $M \hookrightarrow M \vee S^{2 n} \stackrel{\psi}{\longrightarrow} Y$. 
Nous considérons maintenant le composé:

$$
\Omega\left(M \vee S^{6 n-1}\right) \times S^{2 n-1} \stackrel{\mu}{\longrightarrow} \Omega Y \times \Omega Y \stackrel{\nu}{\longrightarrow} \Omega Y
$$

où $\nu$ est la loi de $H$-espace de $\Omega Y$ et $\mu=\Omega\left(g_{3}+g_{2}\right) \times g_{1}$. Pour justifier que $\nu \circ \mu$ est une équivalence d'homotopie, nous en construisons un modèle dans la catégorie des complexes de chaînes:

- $g_{3}+g_{2}$ a pour modèle d'Adams-Hilton

$$
\begin{gathered}
\mathcal{A}\left(g_{3}+g_{2}\right):(T(\beta, \hat{\beta}, \alpha), \partial) \rightarrow(T(a, b), \partial) \\
|\hat{\beta}|=4 n-2 ;|\beta|=4 n-1 ;|\alpha|=6 n-2 ;|a|=2 n-1 ; \partial \beta=k \hat{\beta} ; \\
\partial \alpha=\partial a=0 ; \partial b=k[a, a] ; \mathcal{A}\left(g_{3}+g_{2}\right)(\beta)=b ; \mathcal{A}\left(g_{3}+g_{2}\right)(\hat{\beta})= \\
{[a, a] ; \mathcal{A}\left(g_{3}+g_{2}\right)(\alpha)=[a, b] .}
\end{gathered}
$$

- $\nu$ a pour modèle la multiplication $T(a, b) \otimes T(a, b) \rightarrow T(a, b)$.

- L'injection $(\mathbb{Z}[1 / 2,1 / 3] a, 0) \rightarrow(T(a, b), \partial)$ est un modèle de $g_{1}$.

Nous en déduisons le modèle de complexes de chaînes cherché:

$$
(T(\beta, \hat{\beta}, \alpha), \partial) \otimes(\mathbb{Z}[1 / 2,1 / 3] a, 0) \rightarrow(T(a, b), \partial)
$$

Cette flèche est évidemment surjective. Déterminons, [8], les séries de Poincaré de la source et du but:

$$
\begin{aligned}
P(T(a, b)) & =\frac{1}{1-t^{2 n-1}-t^{4 n-1}} ; \\
P(T(\beta, \hat{\beta}, \alpha) \otimes \mathbb{Z}[1 / 2,1 / 3] a) & =\frac{1}{1-t^{4 n-2}-t^{4 n-1}-t^{6 n-2}} \cdot\left(1+t^{2 n-1}\right) .
\end{aligned}
$$

L'égalité de ces deux séries implique que la flèche est un isomorphisme au niveau des complexes de chaînes, donc induit un isomorphisme en homologie.

Exemple. Notons $f: S^{10 n-3} \rightarrow Y$ le crochet de Whitehead de $S^{6 n-1} \rightarrow Y$ et de $\psi \circ \varphi_{1}: S^{4 n-1} \rightarrow Y$. La Proposition 6 implique en particulier que

$$
S^{2 n} \cup_{k\left[\iota_{2 n}, \iota_{2 n}\right]} e^{4 n} \cup_{f} e^{10 n-2}
$$

est de longueur de cône (et de LS-catégorie) 2.

Démonstration du Théorème 1: Nous allons décomposer cette preuve en 4 étapes qui reprennent un pas de la démonstration du Théorème 
de Hilton-Milnor, telle qu'elle apparaît dans [17]; nous effectuerons des rappels suffisants pour une lecture autonome du texte.

Etape 1: Rappel d'une décomposition utilisée dans la démonstration du Théorème de Hilton-Milnor, [17].

Si $A$ et $B$ sont des $C W$-complexes (pointés), on a une équivalence d'homotopie

$$
\Omega \Sigma(A \vee B) \simeq \Omega \Sigma A \times \Omega \Sigma\left(\bigvee_{i=0}^{\infty} B \wedge A^{(i)}\right)
$$

où $A^{(i)}$ désigne le smash-produit itéré $i$-fois, et $B \wedge A^{(0)}=B$ par convention. Cette équivalence est donnée par une suite d'applications:

$$
v_{n}: B \wedge A^{(n)} \rightarrow \Omega \Sigma(A \vee B) .
$$

Notons $j_{A}$ (resp. $j_{B}$ ) l'injection $A \hookrightarrow A \vee B$ (resp. $B \hookrightarrow A \vee B$ ). On définit $v_{0}$ comme le composé de $\Omega \Sigma\left(j_{B}\right)$ avec la counité de l'adjonction $(\Omega, \Sigma)$ :

$$
v_{0}: B \rightarrow \Omega \Sigma B \rightarrow \Omega \Sigma(A \vee B) .
$$

Supposons avoir construit $v_{n}$, l'application

$$
v_{n+1}: B \wedge A^{(n+1)} \simeq\left(B \wedge A^{(n)}\right) \wedge A \rightarrow \Omega \Sigma(A \vee B)
$$

s'obtient en factorisant par le smash-produit l'application

$$
\left(B \wedge A^{(n)}\right) \times A \stackrel{v_{n} \times w_{0}}{\longrightarrow} \Omega \Sigma(A \vee B) \times \Omega \Sigma(A \vee B) \stackrel{\lfloor,\rangle}{\longrightarrow} \Omega \Sigma(A \vee B),
$$

où $w_{0}: A \rightarrow \Omega \Sigma A \rightarrow \Omega \Sigma(A \vee B)$ est défini de manière analogue à $v_{0}$. Il reste ensuite à prendre la "somme" de ces applications

$$
\tilde{v}=+{ }_{i} v_{i}=\bigvee_{i=0}^{\infty} B \wedge A^{(i)} \rightarrow \Omega \Sigma(A \vee B)
$$

à l'étendre en une application de $H$-espaces

$$
v: \Omega \Sigma\left(\bigvee_{i=0}^{\infty} B \wedge A^{(i)}\right) \rightarrow \Omega \Sigma(A \vee B)
$$

et à composer avec la multiplication $\nu$ de $\Omega \Sigma(A \vee B)$ :

$$
\begin{aligned}
\Omega \Sigma A \times \Omega \Sigma\left(\bigvee_{i=0}^{\infty} B \wedge A^{(i)}\right) \stackrel{\Omega \Sigma j_{A} \times v}{\longrightarrow} \Omega \Sigma(A \vee B) \times \Omega \Sigma(A \vee B) \\
\stackrel{\nu}{\longrightarrow} \Omega \Sigma(A \vee B) .
\end{aligned}
$$


Le fait que ce composé induise une équivalence d'homotopie est le but du "Step IV page 527" de [17] dont ces lignes sont extraites.

Etape 2: L'application $\rho: S^{2 n-1} \wedge M^{\prime} \wedge M^{\prime} \rightarrow S^{2 n-1} \wedge S^{4 n-1} \wedge M^{\prime}$, induite par l'application de pincement $M^{\prime} \rightarrow S^{4 n-1}$, admet une section homotopique.

Soit $M^{\prime}=S^{4 n-2} \cup_{k \iota_{4 n-2}} e^{4 n-1}$, Neisendorfer a exhibé une équivalence d'homotopie [11, Corollary 6.6 p. 36]:

$$
\Delta+i:\left(S^{8 n-4} \cup_{k \iota_{8 n-4}} e^{8 n-3}\right) \vee\left(S^{8 n-3} \cup_{k \iota_{8 n-3}} e^{8 n-2}\right) \rightarrow M^{\prime} \wedge M^{\prime} .
$$

On en déduit que

$$
S^{8 n-3} \cup_{k \iota_{8 n-3}} e^{8 n-2} \rightarrow M^{\prime} \wedge M^{\prime} \rightarrow S^{4 n-1} \wedge M^{\prime}
$$

est une équivalence d'homotopie. Par conséquent, $M^{\prime} \wedge M^{\prime} \rightarrow S^{4 n-1} \wedge M^{\prime}$ et $\rho: S^{2 n-1} \wedge M^{\prime} \wedge M^{\prime} \rightarrow S^{2 n-1} \wedge S^{4 n-1} \wedge M^{\prime}$ ont des sections homotopiques.

Par itération de cet argument, on obtient, pour tout $i \geq 2$, une section homotopique à

$$
S^{2 n-1} \wedge M^{\prime(i)} \wedge\left(S^{2 n-1} \wedge M^{\prime}\right)^{(j)} \rightarrow S^{6 n-2} \wedge M^{\prime(i-1)} \wedge\left(S^{6 n-2}\right)^{(j)} .
$$

Etape 3: Décomposition de la source et du but de $\Omega \psi: \Omega \Sigma\left(M^{\prime} \vee\right.$ $\left.S^{2 n-1}\right) \rightarrow \Omega Y$.

En appliquant l'étape 1, nous obtenons une équivalence d'homotopie:

$$
\begin{aligned}
& \Omega \Sigma\left(M^{\prime} \vee S^{2 n-1}\right) \simeq \Omega \Sigma M^{\prime} \times \Omega \Sigma\left(\bigvee_{i=0}^{\infty} S^{2 n-1} \wedge M^{\prime}(i)\right) \\
& \simeq \Omega \Sigma M^{\prime} \times \Omega \Sigma S^{2 n-1} \\
& \times \Omega \Sigma\left(\bigvee_{i=1, j=0}^{\infty} S^{2 n-1} \wedge M^{\prime(i)} \wedge\left(S^{2 n-1}\right)^{(j)}\right) .
\end{aligned}
$$

La Proposition 6 donne

$$
\begin{aligned}
\Omega Y & \simeq S^{2 n-1} \times \Omega \Sigma\left(M^{\prime} \vee S^{6 n-2}\right) \\
\simeq & S^{2 n-1} \times \Omega \Sigma M^{\prime} \times \Omega \Sigma\left(\bigvee_{i=0}^{\infty} S^{6 n-2} \wedge M^{\prime}(i)\right) \\
\simeq S^{2 n-1} & \times \Omega \Sigma M^{\prime} \times \Omega \Sigma S^{6 n-2} \\
& \left.\times \Omega \Sigma\left(\bigvee_{i=1, j=0}^{\infty} S^{6 n-2} \wedge M^{\prime(i)} \wedge\left(S^{6 n-2}\right)^{(j)}\right)\right)
\end{aligned}
$$


Pour comprendre comment $\Omega \psi$ se comporte relativement à cette décomposition, rappelons de la démonstration de la Proposition 6 que

$$
\begin{aligned}
& \Omega \Sigma M^{\prime} \rightarrow \Omega\left(M \vee S^{2 n}\right) \stackrel{\Omega \psi}{\longrightarrow} \Omega Y \simeq S^{2 n-1} \times \Omega \Sigma\left(M^{\prime} \vee S^{6 n-2}\right) \\
& \rightarrow \Omega \Sigma\left(M^{\prime} \vee S^{6 n-2}\right)
\end{aligned}
$$

est donné par l'injection $M^{\prime} \hookrightarrow M^{\prime} \vee S^{6 n-2}$.

Enfin, le composé de $\Omega \psi$ avec la projection sur $S^{2 n-1} \times \Omega \Sigma M^{\prime}$ ayant un inverse à droite, il nous suffit d'étudier le composé, $\Omega(\psi)^{\prime}$, de $\Omega \psi$ avec la projection canonique:

$$
\begin{aligned}
\Omega(\psi)^{\prime}: \Omega \Sigma\left(M^{\prime} \vee S^{2 n-1}\right) & \stackrel{\Omega \psi}{\longrightarrow} \Omega Y \\
& \left.\rightarrow \Omega \Sigma\left(\bigvee_{i=1, j=0}^{\infty} S^{6 n-2} \wedge M^{\prime(i)} \wedge\left(S^{6 n-2}\right)^{(j)}\right)\right)
\end{aligned}
$$

Etape 4: Construction d'une section homotopique:

$$
\begin{aligned}
\Omega \Sigma\left(\bigvee_{i=1, j=0}^{\infty} S^{6 n-2} \wedge\right. & \left.M^{\prime(i)} \wedge\left(S^{6 n-2}\right)^{(j)}\right) \\
& \rightarrow \Omega \Sigma\left(\bigvee_{i=1, j=0}^{\infty} S^{2 n-1} \wedge M^{\prime(i)} \wedge\left(S^{2 n-1}\right)^{(j)}\right) .
\end{aligned}
$$

Avant d'aborder cette dernière étape, rappelons quelques résultats déjà obtenus:

- L'application $S^{2 n-1} \wedge M^{\prime} \rightarrow \Omega Y$ factorise par l'application de pincement $S^{2 n-1} \wedge S^{4 n-1} \rightarrow \Omega Y$, qui définit la classe d'homotopie construite dans la Proposition 6; i.e. $S^{2 n-1} \wedge S^{4 n-1} \rightarrow \Omega \Sigma\left(M^{\prime} \vee\right.$ $\left.S^{6 n-2}\right) \simeq \Omega\left(M \vee S^{6 n-1}\right)$ est donnée par l'injection $S^{6 n-1} \hookrightarrow M \vee$ $S^{6 n-1}$.

- Sur les éléments $S^{2 n-1} \wedge M^{\prime(i)} \wedge\left(S^{2 n-1}\right)^{(j)}, i \geq 1$, l'application, de but $\Omega \Sigma\left(M^{\prime} \vee S^{6 n-2}\right)$ est déterminée par les crochets de Samelson (étape 1).

Pour démontrer l'étape 4, commençons par fixer $i$ et $j$.

A $Z=S^{6 n-2} \wedge M^{\prime(i)} \wedge\left(S^{6 n-2}\right)^{(j)}$, on associe $Z_{1}=S^{2 n-1} \wedge M^{\prime(i+j+1)} \wedge$ $\left(S^{2 n-1}\right)^{(j)}$. Posons $Z_{2}=S^{2 n-1} \wedge M^{\prime} \wedge M^{\prime} i \wedge\left(M^{\prime} \wedge S^{2 n-1}\right)^{(j)}$; l'associativité et la commutativité du smash-produit fournissent une équivalence 
d'homotopie entre $Z_{1}$ et $Z_{2}$. Il faut noter que cette équivalence modifie le plongement dans $\Omega\left(M \vee S^{2 n}\right)$; le parenthésage des crochets de Samelson est modifié en

$$
\langle\cdots\left\langle j_{1}, j_{2}\right\rangle, \overbrace{\left.\left.j_{2},\right\rangle, \cdots j_{2}\right\rangle}^{i \text { fois }}, \overbrace{\left\langle j_{1}, j_{2}\right\rangle, \cdots\left\langle j_{1}, j_{2}\right\rangle}^{j \text { fois }}\rangle
$$

avec les notations de la démonstration de la Proposition 6. Considérons la restriction $\Omega(\psi)^{\prime \prime}$ de $\Omega(\psi)^{\prime}$ à un sous-bouquet:

$$
\begin{gathered}
\Omega \Sigma\left(\bigvee_{i=1, j=0} S^{2 n-1} \wedge M^{\prime} \wedge M^{\prime(i)} \wedge\left(M^{\prime} \wedge S^{2 n-1}\right)^{(j)}\right) \\
\downarrow \Omega(\psi)^{\prime \prime} \\
\Omega \Sigma\left(\bigvee_{i=1, j=0} S^{6 n-2} \wedge M^{\prime(i)} \wedge\left(S^{6 n-2}\right)^{(j)}\right)
\end{gathered}
$$

Il est bien clair que si $\Omega(\psi)^{\prime \prime}$ a un inverse à droite, il en est de même pour $\Omega(\psi)^{\prime}$. Comme nous l'avons constaté, $\Omega(\psi)^{\prime \prime}$ factorise par les applications de pincement en:

$$
\begin{gathered}
\Omega \Sigma\left(\bigvee_{i=1, j=0} S^{2 n-1} \wedge M^{\prime} \wedge M^{\prime(i)} \wedge\left(M^{\prime} \wedge S^{2 n-1}\right)^{(j)}\right) \\
\qquad \Sigma \mu_{1}\left(\bigvee_{i=1, j=0} S^{2 n-1} \wedge S^{4 n-1} \wedge M^{\prime(i)} \wedge\left(S^{4 n-1} \wedge S^{2 n-1}\right)^{(j)}\right) \\
\left.\qquad \downarrow^{\mu_{2}}\right) \\
\quad \Omega \Sigma\left(\bigvee_{i=1, j=0} S^{6 n-2} \wedge M^{\prime(i)} \wedge\left(S^{6 n-2}\right)^{(j)}\right) .
\end{gathered}
$$

Le résultat annoncé se déduit maintenant de:

- l'application $\mu_{1}$ admet un inverse à droite d'après l'étape 2;

- l'application $\mu_{2}$ est un isomorphisme; ce dernier point provient de la connaissance de $\Omega(\psi)^{\prime}$ et de la modification de parenthésage entre $Z_{1}$ et $Z_{2}$. 


\section{Démonstration du Théorème 2}

Rappelons, [16], que si $Z$ est un CW-complexe simplement connexe de $R$-localisé $Z_{R}$, alors cat $Z_{R} \leq$ cat $Z$.

Notons $e_{R}^{n}$ le cône construit sur la sphère $R$-locale $S_{R}^{n-1}$. Soit $X=$ $S_{R}^{n_{1}} \cup_{f_{1}} e_{R}^{n_{2}} \cup_{f_{2}} e_{R}^{n_{3}}, n_{1} \leq n_{2} \leq n_{3}$, une décomposition $R$-cellulaire de $X$. L'attachement d'un cône augmentant la catégorie d'au plus une unité, il faut que $X^{\prime}=S_{R}^{n_{1}} \cup_{f_{1}} e_{R}^{n_{2}}$ soit de LS-catégorie 2 .

Jusqu'à la dimension $m$, les groupes d'homotopie de la sphère $R$-locale, $S_{R}^{n_{1}}$, s'injectent dans les groupes d'homotopie du rationalisé $S_{\mathbb{Q}}^{n_{1}}$. La seule possibilité pour que $X^{\prime}$ ne soit pas une suspension est donc $n_{1}=2 n$ et $f_{1}=k\left[\iota_{2 n}, \iota_{2 n}\right]$, soit, avec les notations de la Proposition $6, X^{\prime}=Y_{R}$. Considérons maintenant l'attachement de la dernière cellule:

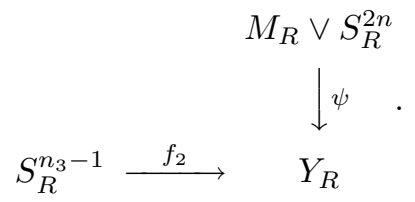

Si $f_{2} \in \operatorname{Im} \pi_{*}(\psi)$, notons $\tilde{f}_{2}: S_{R}^{n_{3}-1} \rightarrow M_{R} \vee S_{R}^{2 n}$ un relevé de $f_{2}$. Il est alors facile de constater que $X$ est un 2-cône, (donc de LS-catégorie $\leq 2$ ):

$$
S_{R}^{n_{3}-1} \vee S_{R}^{4 n-1} \stackrel{g}{\rightarrow} M_{R} \vee S_{R}^{2 n} \rightarrow X
$$

où $g=\left(\varphi_{1}-\varphi_{2}\right)+\tilde{f}_{2}$.

Pour avoir un espace $X$ de LS-catégorie 3, la seule possibilité est donc que la classe d'homotopie $f_{2}$ soit de la forme $l[a, b]+\mu, l \neq 0$, $\mu \in \operatorname{Im} \pi_{*}(\psi)$, où $[a, b]$ est le crochet relatif représenté par $S_{R}^{6 n-1} \rightarrow Y_{R}$ et décrit dans la démonstration de la Proposition 6 . Le cocycle associé à $S_{R}^{n_{1}}$ dans la cohomologie de $X$ a une puissance troisième non nulle, car égale à $l k\left(e_{R}^{n_{3}}\right)^{*}$, où $\left(e_{R}^{n_{3}}\right)^{*}$ désigne la classe de cohomologie associée à $e_{R}^{n_{3}}$.

Remarque. En rationnel, il est assez facile de voir que le résultat du Théorème 2 s'étend aux CW-complexes de LS-catégorie $k$ admettant une décomposition en $k$ cellules rationnelles; cette propriété est-elle conservée en $R$-local?

i.e.: si $X \in C W_{r}^{m}$ est un CW-complexe de LS-catégorie $k$, admettant une décomposition en $k$ cellules $R$-locales, son algèbre de cohomologie admet-elle un cup-produit de longueur $k$ ? 


\section{Un exemple tempéré}

Dans cette section, tous les espaces considérés sont localisés en dehors de 2 et de 3 .

Soit $Y=S^{6} \cup_{\left[\iota_{6}, \iota_{6}\right]} e^{12}$, on sait que $\Omega Y$ a le type d'homotopie de $S^{5} \times$ $\Omega\left(S^{17}\right)$, (cf Proposition 6). Notons $\rho: S^{17} \rightarrow Y$ la classe d'homotopie correspondant à cette décomposition et $\beta$ le générateur d'ordre 5 de $\pi_{24}\left(S^{17}\right)$.

Proposition 7. Le $C W$-complexe $Z=Y \cup_{\rho \circ \beta} e^{25}$ est de LS-catégorie 3 , sa longueur de cup-produit est 2 .

Remarque. Dans [2], [3], D. Anick introduit une généralisation des espaces $R$-locaux, les espaces tempérés, ("mild space"). Notons $\bar{p}$ le plus petit entier non inversible dans $R$; dans le cadre tempéré, la catégorie $C W_{r}^{m}$ est remplacée par la catégorie $C W_{r}^{q}$ des $C W$-complexes $R$-locaux, $r$-réduits et de $R$-dimension $\leq q$ avec $q=(r-1) \bar{p}$.

Remarquons que $Z$ est 6-réduit, de dimension 25; on peut donc choisir 5 comme premier entier non inversible dans $R$. L'espace $Y$ est donc tempéré au sens ci-dessus.

Démonstration: Pour justifier l'affirmation sur la LS-catégorie, considérons l'espace, $V=Y \cup_{\rho} e^{18}$, également obtenu comme cofibre $S^{17} \cup_{\beta}$ $e^{25} \rightarrow Z \rightarrow V$, ainsi que le montre le diagramme:

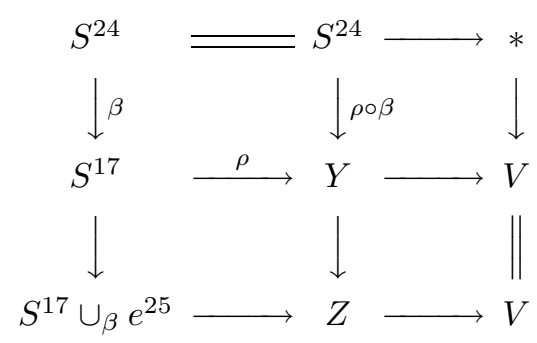

dans lequel la ligne du bas et la colonne de droite sont constituées des cofibres. Notons:

- $p_{2, Z}: E_{2} Z \rightarrow Z$ et $p_{2, V}: E_{2} V \rightarrow V$ les fibrations de Ganea associées à $Z$ et $V$ respectivement,

- $E_{2} Z \rightarrow Z \stackrel{\tau_{Z}}{\longrightarrow} Z / E_{2} Z$ et $E_{2} V \rightarrow V \stackrel{\tau_{V}}{\longrightarrow} V / E_{2} V$ les cofibrations associées à $p_{2, Z}$ et $p_{2, V}$ respectivement. 
Si nous montrons que l'application $\Sigma \tau_{Z}$ n'est pas homotopiquement triviale, il est alors facile de constater que $p_{2, Z}$ n'a pas de section homotopique, (i.e. cat $Z \geq$ conil $Z \geq 3$ ). Pour cela, il nous suffit de démontrer que le composé

$$
\Sigma Z \rightarrow \Sigma V \stackrel{\Sigma \tau_{V}}{\longrightarrow} \Sigma\left(V / E_{2} V\right)
$$

n'est pas homotopiquement trivial, car il factorise par $\Sigma \tau_{Z}: \Sigma Z \rightarrow$ $\Sigma\left(Z / E_{2} Z\right)$.

Si $W$ est un CW-complexe, notons $W_{[k]}$ son squelette de dimension $k$ et remarquons que pour le problème étudié, il suffit de déterminer $E_{2}(V)_{[26]}$. Avec un raisonnement analogue à celui effectué pour décomposer $\Omega \mathbb{C P}^{3}$, on obtient $\Omega V=S^{5} \times \Omega\left(S^{23}\right)$, d'où l'on déduit:

$$
\begin{aligned}
& (\Sigma \Omega V)_{[26]}=S^{6} \vee S^{23} \\
& (\Omega V * \Omega V)_{[26]}=S^{11} \\
& \left(E_{2} V\right)_{[26]}=\left(S^{6} \cup_{\left[\iota_{6}, \iota_{6}\right]} e^{12}\right) \vee S^{23}=Y \vee S^{23} .
\end{aligned}
$$

La flèche

$\Sigma Z=S^{7} \vee S^{13} \vee S^{26} \rightarrow \Sigma V=S^{7} \vee S^{13} \vee S^{19} \rightarrow\left(\Sigma\left(V / E_{2} V\right)\right)_{[27]}=S^{19} \vee S^{24}$

"contient" $\Sigma^{2} \beta: S^{26} \rightarrow S^{19}$ qui n'est pas homotopiquement triviale.

\section{Références}

1. F. Adams And P. Hilton, On the chain algebra of a loop space, Comment. Math. Helv. 30 (1956), 305-330.

2. D. Anick, Hopf algebras up to homotopy, J. Amer. Math. Soc. 2 (1989), 417-543.

3. D. AnICK, R-local homotopy theory, in "Homotopy Theory and Related Topics," Proceedings of the International Conference held in Kinosaki, August 19-24, 1988 (M. Mimura, ed.), Lecture Notes in Math. 1418, Springer-Verlag, 1990, pp. 78-85.

4. I. Berstein And P. Hilton, Category and generalized Hopf invariants, Illinois J. Math. 4 (1960), 437-451.

5. W. G. Dwyer, Tame homotopy theory, Topology 18 (1979), 321338.

6. T. GaneA, Lusternik-Schnirelmann category and strong category, Illinois J. Math. 11 (1967), 417-427.

7. I. M. JAMES, On category in the sense of Lusternik-Schnirelmann, Topology 17 (1978), 331-348. 
8. J.-M. Lemaire, "Algèbres connexes et Homologie des espaces de lacets," Lecture Notes in Math. 422, Springer-Verlag, 1974.

9. J.-M. Lemaire, Lusternik-Schnirelmann category: an introduction, in "Algebra, Algebraic Topology and their Interactions," Proceedings of a conference held in Stockholm, August 3-13, 1983, and later developments (J.-E. Roos, ed.), Lecture Notes in Math. 1183, 1986, pp. 259-276.

10. L. Lusternik And L. Schnirelmann, "Méthodes topologiques dans les problèmes variationnels," Hermann, Paris, 1934.

11. J. Neisendorfer, Primary homotopy theory, Mem. Amer. Math. Soc. 232 (1980), 67 pp.

12. J. Neisendorfer ANd P. Selick, Some examples of spaces with or without exponents, in "Current trends in Algebraic Topology, Part 1," (London, Ont., 1981), CMS Conf. Proc. 2, Amer. Math. Soc., 1982, pp. 343-357.

13. H. Scheerer and D. Tanré, R-local homotopy theory, Bull. London Math. Soc. 22 (1990), 591-598.

14. P. Schweitzer, Secondary cohomology operations induced by the diagonal mapping, Topology 3 (1965), 337-355.

15. W. Singhof, Generalized higher order cohomology operations induced by the diagonal mapping, Math. Z. 162 (1978), 7-26.

16. G. H. Toomer, Topological localization, category and cocategory, Canad. J. Math. 27 (1975), 319-322.

17. G. W. Whitehead, "Elements of homotopy theory," Springer Verlag GTM 61, 1978.

\author{
Hans Scheerer: \\ Freie Universität Berlin \\ Mathematisches Institut \\ Arnimallee 2-6 \\ D-14195 Berlin \\ ALLEMAGNE
}

\author{
Daniel Tanré: \\ U.R.A. CNRS 0751 \\ U.F.R. de Mathématiques \\ Université des Sciences et Technologies \\ 59655 Villeneuve d'Ascq Cedex \\ FRANCE
}

Primera versió rebuda el 2 de Setembre de 1996, darrera versió rebuda el 18 de Febrer de 1997 